\title{
Dark matter annihilation into right-handed neutrinos and the galactic center gamma-ray excess
}

\author{
Yi-Lei Tang ${ }^{a}$ and Shou-hua Zhu ${ }^{a, b, c}$ \\ ${ }^{a}$ Center for High Energy Physics, Peking University, \\ Beijing 100871, China \\ ${ }^{b}$ Institute of Theoretical Physics \& State Key Laboratory of Nuclear Physics and Technology, \\ Peking University, Beijing 100871, China \\ ${ }^{c}$ Collaborative Innovation Center of Quantum Matter, \\ Beijing 100871, China \\ E-mail: tangyilei15@pku.edu.cn, shzhu@pku.edu.cn
}

\begin{abstract}
In this paper, we will discuss a specific case that the dark matter particles annihilate into right-handed neutrinos. We calculate the predicted gamma-ray excess from the galactic center and compare our results with the data from the Fermi-LAT. An approximately $10-60 \mathrm{GeV}$ right-handed neutrino with heavier dark matter particle can perfectly explain the observed spectrum. The annihilation cross section $\langle\sigma v\rangle$ falls within the range $0.5-4 \times 10^{-26} \mathrm{~cm}^{3} / \mathrm{s}$, which is roughly compatible with the WIMP annihilation cross section.
\end{abstract}

Keywords: Beyond Standard Model, Neutrino Physics

ARXiv EPrint: 1512.02899 


\section{Contents}

1 Introduction 1

2 Right-handed neutrino models 2

3 Simulations and numerical results 3

4 Summary and conclusions $\quad 6$

\section{Introduction}

The indirect detection experiments of the dark matter (DM) focus on observing the standard model $(\mathrm{SM})$ products from the annihilation or decay of the dark matter particles. Various analyses of the Fermi-LAT data reveal an excess in $1-4 \mathrm{GeV}$ gamma rays from near the center of the milky way [1-12]. Fittings to the excess have been carried out (For some examples, see ref. [10, 13-20]) by assuming that the dark matter particles mainly annihilate into $b \bar{b}, \tau \bar{\tau}, W^{+} W^{-}, Z Z, h h, t \bar{t}$ (For examples, see ref. [10, 13]). Constraints from the dwarf spheroidal (dSph) galaxy candidates are given in ref. [21-28]. However, interestingly, there seem to be slight gamma signals from two of the dSphs recently [2932]. Cascade annihilations sometimes appear in the literature in order to avoid the direct detection bounds (For examples, see ref. [33-44]). Among all these channels, the $b \bar{b}$ channel and the $h h$ offer better-fitted spectrum. The spectrum predicted by the $W^{+} W^{-}$and the $Z Z$ channels usually peak in relatively higher energy-scales thus being less favoured.

Besides these fully discussed channels, in the literature, models in which the dark matter particles might mainly annihilate into light right-handed neutrinos do exist. For example, the next to minimal supersymmetric standard model (NMSSM) can be extended with right-handed neutrino superfield(s) which only couple(s) with the singlet Higgs [34, 4551]. Some papers have discussed about the right-handed sneutrino dark matter annihilating into $b \bar{b}$, exotics Higgs pairs. However, there does exist some parameter space that the dark matter particles mainly annihilate into the right-handed neutrinos. Ref. [52-55] also proposed a model in which dark matter particles mainly annihilate into the right-handed neutrinos through t-channel $Z^{\prime}$ mediators.

In the type I see-saw mechanisms [56-60], right-handed neutrinos slightly mix with the SM light neutrinos and mainly decay through $h / Z+\nu_{e, \mu, \tau}, W^{ \pm}+l^{\mp}$ channels by the mixing with the SM neutrinos. If the dark matter particles near the galactic center mainly annihilate into the right-handed neutrinos, these right-handed neutrinos then decay and finally produce the gamma-ray photons detected by the Fermi-LAT. If the mass of the righthanded neutrino $m_{N} \gtrsim 100 \mathrm{GeV}$, it will mainly decay into on-shell $W / Z$ bosons, raising the position of the peak value. In fact, a simple simulation by the micrOMEGAs [61] shows 
that in such cases, the predicted gamma spectrum are no better then those in the $W^{+} W^{-}$ and $Z Z$ cases.

If the mass of the right-handed neutrino is less then $m_{W}$, which is just the case to be discussed in the following text, it will decay through off-shell $h / Z / W^{ \pm}$bosons. In order to deal with the off-shell $W / Z / H$ cases, we use MadGraph5_aMC@NLO2.3.2 [62] to generate the three-body decay events of one right-handed neutrino at rest in the parton-level, and then input the event file into PYTHIA 8.212 [63] to do parton-shower, hadronization, decay process and finally boost the photon spectrum in order to compare the gamma-ray spectrum with the one from ref. [12].

\section{Right-handed neutrino models}

If the dark matter particles $\chi$ with the mass $m_{\chi}$ mainly annihilate into right-handed neutrinos $N$, the key to acquire the gamma-ray spectrum is to calculate the decay processes of the $N$. We input the following Lagrangian into FeynRules 2.3 [64],

$$
\mathcal{L} \supset \frac{1}{2} \bar{N} \gamma^{\mu} \partial_{\mu} N-\frac{1}{2} m_{N} \bar{N} N-\left(y_{i} \bar{l}_{L i} \cdot \tilde{H} N+\text { h.c. }\right),
$$

where $m_{N}$ is the mass of the right-handed neutrino. $l_{L i}$ 's are the left-handed leptonic $\mathrm{SU}(2)_{L}$ doublets. $i$ runs from $1-3$ to indicate the $e, \mu, \tau$ generation. $\tilde{H}=i \tau_{2} H^{*}$ is the SM Higgs doublet field, where $\tau_{2}$ is one of the Pauli matrix. Although in the standard type-I see-saw mechanisms, more than one right-handed neutrinos are needed in order to generate a complete neutrino mass spectrum, in this paper, we assume that only one righthanded neutrino is lighter then the dark matter particle for simplicity. Therefore, it can be produced on-shell during the annihilation processes. If one would like to discuss the cases of more than one right-handed neutrinos, he could just linearly combine the spectrum corresponding with each right-handed neutrino. Thus, eq. (2.1) can summarize the features of the right-handed neutrinos in most of the right-handed neutrino models. After the Higgs field gets a vacuum expectation value (vev),

$$
\langle H\rangle=\left[\begin{array}{l}
0 \\
v
\end{array}\right],
$$

where $v=174 \mathrm{GeV}$, the last term in (2.1) introduces tiny mixing between $N$ and the SM neutrinos, resulting in effective $N-l_{i}^{ \pm}-W^{\mp}, N-\nu_{i}$ - $Z$ vertices through mixing between $N$ and $\nu_{i}$,

$$
\mathcal{L} \supset c g_{2} \sqrt{2} \theta_{i}\left(W_{\mu}^{+} \bar{N} \gamma^{\mu} P_{L} l_{i}^{-}+\text {h.c. }\right)+\frac{g_{2}}{\cos \theta_{W}} \theta_{i} Z_{\mu}\left(\bar{N} \gamma^{\mu} P_{L} \nu_{i}+\text { h.c. }\right)
$$

where

$$
\theta_{i} \approx \frac{y_{i} v}{m_{N}}
$$

are the mixing parameters, $g_{2}=\sqrt{4 \sqrt{2} G_{F} m_{W}^{2}}$ is the weak-coupling constant, and $\theta_{W}$ is the Weinberg angle. Note that (2.4) are the first-order results calculated by the perturbation 
theory of diagonalizing the matrices. As we have noted in the previous section, we only discuss the $m_{N}<m_{W}$ case in the following text of this paper, so the effective $N-W^{ \pm} l^{\mp}$ and $N-Z-\nu_{e, \mu, \tau}$ coupling constants are similar with the $N-H-\nu_{e, \mu, \tau}$ coupling constants $y_{i}$. However, compared with the $W$ and $Z$ bosons, the coupling constants between the SMHiggs and other light SM fermions are quite small, and all the mediators $Z / W^{ \pm} / h$ are off-shell when the right-handed neutrino $N$ decays, so the processes $N \rightarrow h^{*} \nu_{i} \rightarrow$ all $+\nu_{i}$ are negligible. Although the constants $\frac{y_{i}}{m_{N}}$ decide the total width of $N$, from (2.3), (2.4) we can learn that the ratio between the $Z$ and $W^{ \pm}$coupling constants are not affected. That is to say, for each $i=1,2,3$, the ratios $\frac{B r\left(N \rightarrow \nu_{i} Z^{*}\right)}{B r\left(N \rightarrow l_{i}^{ \pm} W^{\mp *}\right)}$ are fixed if only a $m_{N}$ is determined.

Assuming that the SM-neutrino masses originate from the Type-I see-saw mechanisms, the mixing parameters $\theta_{i} \approx \frac{y_{i} v}{m_{N}}$ can be large enough so that the lifetime of the righthanded neutrino can be short enough if only $m_{N} \gtrsim 1 \mathrm{GeV}$. According to the oscillation data [65], at least one light neutrino should be heavier than $0.1 \mathrm{eV}$ which means at least one $\frac{y_{i}^{2} v^{2}}{m_{N}}>0.1 \mathrm{eV}$. If, for example, $m_{N}=5 \mathrm{GeV}$, the mixing parameter $\theta_{i}=\frac{y_{i} v}{m_{N}}>3 \times 10^{-6}$, which leaves more than enough room beyond the ability of the searching proposals on colliders or other techniques (For some recent experimental and theoretical works on this topic, see ref. [66-74]). Simple simulations by MadGraph also show that $\tau_{N} \lesssim 10^{-3}$ sec $\ll$ $1 \mathrm{sec}$, which means once produced, the right-handed neutrinos decay immediately before travelling too far away from the galactic center where they are produced. Further more, we know there are models [75-79] that can reach larger $\theta_{i}$ while keeping light neutrino masses to be small.

\section{Simulations and numerical results}

In this paper, we ignore the inverse Compton, the synchrotron, and the bremsstrahlung emissions from the charged particle products. We only consider the photons emitted during the showering processes and from the decays of the hadrons. Since the decays of the tau leptons produce photons while the electrons and muons do not, we only discuss the following two scenarios for simplicity,

- $y_{1}=y_{2}=0, y_{3} \neq 0$. Then $100 \%$ of the right-handed neutrinos decay through $\tau+W^{*} / \nu_{\tau}+Z^{*}$ channels. The tau leptons also contribute to the gamma-ray spectrum.

- $y_{3}=0, y_{1}^{2}+y_{2}^{2} \neq 0$. Since muons and electrons do not produce photons, and the ratios $\frac{B r\left(N \rightarrow \nu_{e} Z^{*}\right)}{B r\left(N \rightarrow l_{e}^{ \pm} W^{\mp *}\right)}=\frac{B r\left(N \rightarrow \nu_{\mu} Z^{*}\right)}{B r\left(N \rightarrow l_{\mu}^{ \pm} W^{\mp *}\right)}$ are fixed at a given $m_{N} \gg m_{\mu}$, the gamma-ray spectrum should be independent on concrete values of $y_{1,2}$.

The gamma-ray spectrum by general values of $y_{1,2,3}$ are just linear-combinations of the above two cases.

Since we are discussing a pair of dark matter particles $\chi$ annihilating into a pair of right-handed neutrinos $N, m_{\chi} \geq m_{N}$ should be satisfied. If $m_{\chi}>m_{N}$, the spectrum will also be boosted. The dark matter profile and the $\langle\sigma v\rangle$ also affect the height of the spectrum. In this paper, in order to compare our results with the ref. [12, 13], we adopt 
the Navarro-Frenk-White (NFW) profile [80],

$$
\rho(r)=\rho\left(r_{0}\right) \frac{\left(r / r_{s}\right)^{-\gamma}}{\left(1+r / r_{s}\right)^{3-\gamma}},
$$

where we adopt $r_{s}=20 \mathrm{kpc}, \gamma=1.2$, and $\rho_{0}$ is set in order for the local dark matter density $\rho_{\odot}$ to be $0.4 \mathrm{GeV} / \mathrm{cm}^{3}$ at $r_{\odot}=8.5 \mathrm{kpc}$. The differential flux of the photons from a given direction $\psi$ is given by

$$
\frac{d N}{d \Omega d E}(\psi)=\frac{1}{4 \pi \eta} \frac{J(\psi)}{m_{\chi}^{2}}\langle\sigma v\rangle \frac{d N}{d E_{\gamma}},
$$

with $\eta=2(4)$ for the self-conjugate (non-self-conjugate) dark matter. For simplicity and without loss of generality, we adopt $\eta=2$ in this paper. $\frac{d N}{d E}$ indicates the spectrum of photons emitted per annihilation process. The definition of $J(\psi)$ is given by

$$
J(\psi)=\int_{\text {l.o.s }} d s \rho(r)^{2}
$$

which is the line-of-sight integral. We use the data integrated within the region of interest (R.O.I) at galactic latitudes $2^{\circ} \leq|b| \leq 20^{\circ}$ and the galactic longitudes $|l|<20^{\circ}$. The averaged $\bar{J}$ is then to become

$$
\bar{J}=\frac{1}{\Delta \Omega} \int_{\Delta \Omega} J(\psi) d \Omega
$$

$\bar{J}$ is calculated to be $2.0 \times 10^{23} \mathrm{GeV}^{2} / \mathrm{cm}^{5}$. More realistically, any modifications to the above profile parameters will result in another $\bar{J}_{\text {real }}$. Define

$$
\bar{J}_{\text {real }}=\mathcal{J} \times \bar{J}
$$

then

$$
\langle\sigma v\rangle=\frac{\langle\sigma v\rangle_{\text {real }}}{\mathcal{J}},
$$

where $\langle\sigma v\rangle_{\text {real }}$ is the modified annihilation cross section in this case.

Since there are large correlations among the systematic errors of different bins in the Calore, Cholis and Weniger's (CCW) fit from the ref. [12], the $\chi^{2}$ should be defined as

$$
\chi^{2}=\left[\frac{d N}{d E}-\left(\frac{d N}{d E}\right)_{\mathrm{obs}}\right] \cdot \Sigma^{-1} \cdot\left[\frac{d N}{d E}-\left(\frac{d N}{d E}\right)_{\mathrm{obs}}\right] .
$$

We have scanned the $m_{N}-m_{\chi}$ parameter space by a $0.2 \mathrm{GeV}$ interval. For each point in the parameter space, we used MadGraph5_aMC@NLO2.3.2 to generate an one-millionevent sample file. Then we sent these events to PYTHIA 8.212 in order to acquire the photon spectrum. This process is most time-consuming during the calculations. We list the 1,2 and $3 \sigma$ area in the figure 1 . The best-fitted points are $m_{N}=32.0 \mathrm{GeV}, m_{\chi}=44.2 \mathrm{GeV}$, with $\chi^{2}=24.22$ and the best-fitted $\langle\sigma v\rangle=2.63 \times 10^{-26} \mathrm{~cm}^{3} / \mathrm{s}$ for the $y_{1}=y_{2}=0$, $y_{3} \neq 0$ case, and $m_{N}=27.0 \mathrm{GeV}, m_{\chi}=45.4 \mathrm{GeV}$, with $\chi^{2}=23.81$ and the best-fitted 

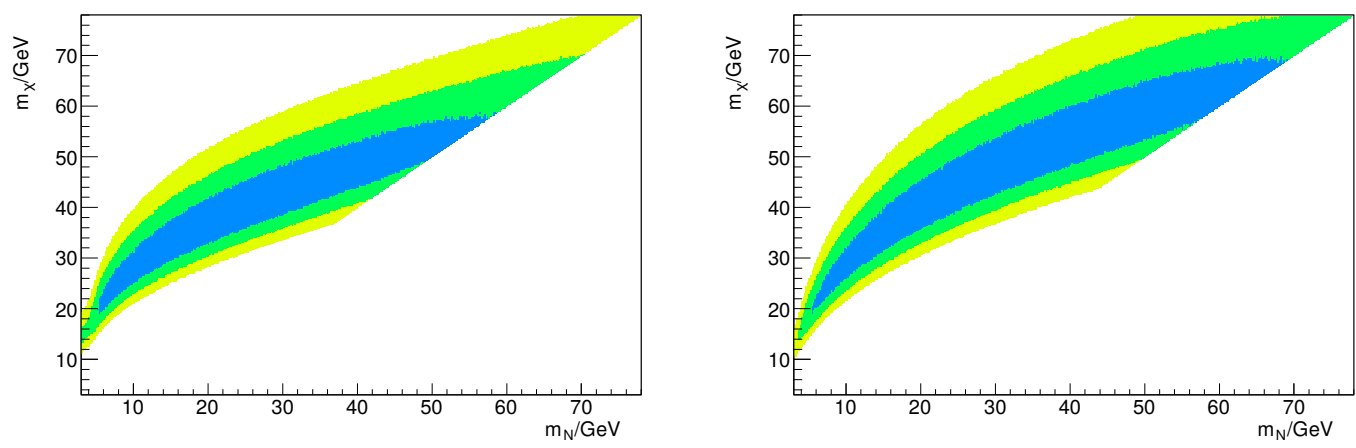

Figure 1. The $\Delta \chi^{2}$ figures. The blue, green, yellow areas are corresponding to the 1,2 and $3 \sigma$ areas respectively. $\langle\sigma v\rangle$ is adjusted in order to acquire the best-fitted result. The left panel indicates the $y_{1}=y_{2}=0, y_{3} \neq 0$ case. The right-panel indicates the $y_{3}=0, y_{1}^{2}+y_{2}^{2} \neq 0$ case.
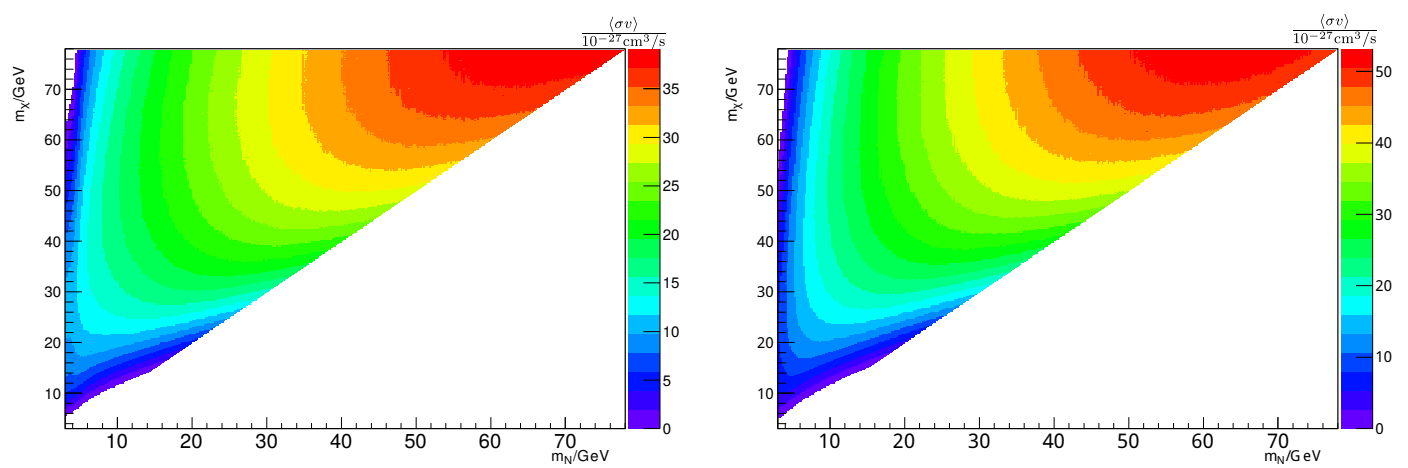

Figure 2. The best-fitted $\langle\sigma v\rangle=\langle\sigma v\rangle_{\text {real }} \mathcal{J}$, in the unit of $\mathrm{cm}^{3} / \mathrm{s}$. The left panel indicates the $y_{1}=y_{2}=0, y_{3} \neq 0$ case. The right-panel indicates the $y_{3}=0, y_{1}^{2}+y_{2}^{2} \neq 0$ case.

$\langle\sigma v\rangle=3.37 \times 10^{-26} \mathrm{~cm}^{3} / \mathrm{s}$ for the $y_{3}=0, y_{1}^{2}+y_{2}^{2} \neq 0$ case. Note that for the $m_{N}<10 \mathrm{GeV}$ cases, which are too near to the $\Lambda_{\mathrm{QCD}}$ scale, the showering and hadronization process from PYTHIA are suspectable. Nevertheless, in the figure 1, 2, we still show our numerical results in this area. However, since the " $1-\sigma$ " area is such a long belt ranging from $10 \mathrm{GeV}$ to $60 \mathrm{GeV}$, the main features of our conclusions should not be affected severely by the uncertainty of the QCD calculations.

In the figure 2, we also plot the best-fitted $\langle\sigma v\rangle$ for each $m_{N}$ and $m_{\chi}$.

From the figure 1, 2, and 3 we can learn that $m_{N}$ approximately ranges from $10 \mathrm{GeV}$ to $60 \mathrm{GeV}$ within $1 \sigma$ level and necessary boost is needed for the best-fitting with the observed excess. Both the $y_{1}=y_{2}=0, y_{3} \neq 0$, and $y_{3}=0, y_{1}^{2}+y_{2}^{2} \neq 0$ show us no significant difference between them. However, a slightly larger $\langle\sigma v\rangle$ is needed in the $y_{3}=0, y_{1}^{2}+y_{2}^{2} \neq 0$ case. This is because the tau leptons produced in the $y_{3} \neq 0$ case strengthen the gamma-ray flux, thus weaken the needed $\langle\sigma v\rangle$ in this case.

Since the gamma-ray signals from near the center of our galaxy are severely contaminated, it is quite important to compare the results with the constraints from the dSph galaxies. Detailed analyses depend closely on the shapes and the fluxes of the spectrum in specific models (For an example of the method, see ref. [81]). In this paper, we only note 


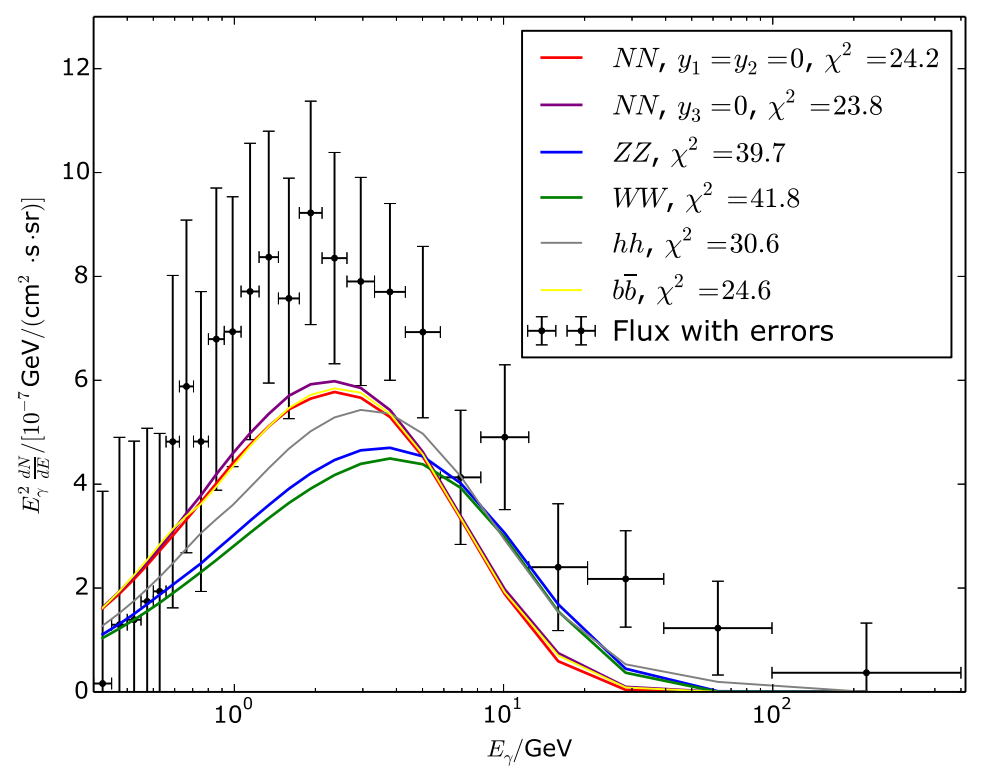

Figure 3. The best-fitted gamma-ray spectrum together with the observed central values and the errorbars. In the case of $y_{1}=y_{2}=0, y_{3} \neq 0, \chi^{2}=24.22$, with the p-value 0.336 . In the case of $y_{3}=0, y_{1}^{2}+y_{2}^{2} \neq 0, \chi^{2}=23.81$, with $\mathrm{p}$-value 0.357 . The data together with the error bars are from ref. [12]. We also plot the gamma-ray spectrum and list the $\chi^{2}$ value of the best-fitted $Z Z$, $W W, h h$, and $b \bar{b}$ channels for comparison. All these curves and values are calculated by a similar MadGraph5_aMC@NLO2.3.2+PYTHIA 8.212 process.

that from observing the spectrum depicted in figure 3, the best-fitted spectrum induced from the right-handed neutrinos are fairly close to the ones induced from the $b \bar{b}$ channel which can help us roughly infer the situation of the right-handed neutrino. According to the ref. [27], the best-fitted $b \bar{b}$ point is located just slightly above the constraint line, which is a subtle case. However, the annihilation rate $\langle\sigma v\rangle_{\text {real }}=\mathcal{J}\langle\sigma v\rangle$ is quite sensitive to the profile parameters, where relatively large uncertainties remain [82]. For example, if the local dark matter density $\rho_{\odot}$ varies from $0.2 \mathrm{GeV} / \mathrm{cm}^{3}$ to $0.6 \mathrm{GeV} / \mathrm{cm}^{3},\langle\sigma v\rangle_{\text {real }}$ can differ by one order of magnitude since it depends on $\rho_{\odot}^{2}$. In this sense, the results from near our galactic center and the constraints from the dSph galaxies are still compatible at present stage.

\section{Summary and conclusions}

In this paper, we calculated the predicted gamma-ray excess from the galactic center in a specific case that dark matter particles annihilate into two light right-handed neutrinos. We find that the $m_{N}$ can range from approximately $10 \mathrm{GeV}$ to $60 \mathrm{GeV}$ within $1 \sigma$ level and necessary boost is needed. The $\langle\sigma v\rangle$ can vary from $0.5 \times 10^{-26} \mathrm{~cm}^{3} / \mathrm{s}$ to $5 \times 10^{-26} \mathrm{~cm}^{3} / \mathrm{s}$, which is roughly compatible with the WIMP annihilation cross section $\langle\sigma v\rangle_{\text {decouple }}=2-3 \times$ $10^{-26} \mathrm{~cm}^{3} / \mathrm{s}$ when the dark matter particles decouple. Comparing the two panels in the figure 2 , we can see that we need a slightly larger $\langle\sigma v\rangle$ in the $y_{3}=0, y_{1}^{2}+y_{2}^{2} \neq 0$ case, since the tau leptons produced in this case is much less then the $y_{3} \neq 0$ case. 
In this paper, we extract some common points from some specific new physics models in which the DM might annihilate mainly into right-handed neutrinos. The detailed properties of the dark matter particles are not discussed in this paper, and the right-handed neutrinos are so weakly coupled with the SM sectors, so it is hardly possible for us to test this scenario by other ways, e.g., collider physics. However, in these specific models in which this scenario is embedded, such right-handed neutrinos can be produced through other mediators. For example, in the NMSSM extended with the right-handed neutrino(s), the exotic singlet-like Higgs boson might decay into the right-handed neutrinos. Detailed discussions about these models in this case shall also be our next topics.

\section{Acknowledgments}

We would like to thank Ran Ding, Jia-Shu Lu, Weicong Huang, Chen Zhang, Weihong Zhang, Yu-Feng Zhou, Lei Feng for helpful discussions. This work was supported in part by the Natural Science Foundation of China (Grants No. 11135003 and No. 11375014).

Open Access. This article is distributed under the terms of the Creative Commons Attribution License (CC-BY 4.0), which permits any use, distribution and reproduction in any medium, provided the original author(s) and source are credited.

\section{References}

[1] L. Goodenough and D. Hooper, Possible evidence for dark matter annihilation in the inner Milky Way from the Fermi Gamma Ray Space Telescope, arXiv:0910.2998 [INSPIRE].

[2] D. Hooper and L. Goodenough, Dark matter annihilation in the galactic center as seen by the Fermi Gamma Ray Space Telescope, Phys. Lett. B 697 (2011) 412 [arXiv:1010.2752] [INSPIRE].

[3] A. Boyarsky, D. Malyshev and O. Ruchayskiy, A comment on the emission from the Galactic Center as seen by the Fermi telescope, Phys. Lett. B 705 (2011) 165 [arXiv:1012.5839] [INSPIRE].

[4] D. Hooper and T. Linden, On the origin of the $\gamma$-rays from the galactic center, Phys. Rev. D 84 (2011) 123005 [arXiv:1110.0006] [INSPIRE].

[5] T. Linden, E. Lovegrove and S. Profumo, The morphology of hadronic emission models for the gamma-ray source at the galactic center, Astrophys. J. 753 (2012) 41 [arXiv:1203.3539] [INSPIRE].

[6] K.N. Abazajian and M. Kaplinghat, Detection of a gamma-ray source in the galactic center consistent with extended emission from dark matter annihilation and concentrated astrophysical emission, Phys. Rev. D 86 (2012) 083511 [Erratum ibid. D 87 (2013) 129902] [arXiv: 1207.6047] [INSPIRE].

[7] D. Hooper and T.R. Slatyer, Two emission mechanisms in the Fermi bubbles: a possible signal of annihilating dark matter, Phys. Dark Univ. 2 (2013) 118 [arXiv:1302.6589] [INSPIRE]. 
[8] C. Gordon and O. Macias, Dark matter and pulsar model constraints from Galactic Center Fermi-LAT gamma ray observations, Phys. Rev. D 88 (2013) 083521 [arXiv:1306.5725] [INSPIRE].

[9] K.N. Abazajian, N. Canac, S. Horiuchi and M. Kaplinghat, Astrophysical and dark matter interpretations of extended gamma-ray emission from the Galactic Center, Phys. Rev. D 90 (2014) 023526 [arXiv: 1402.4090] [INSPIRE].

[10] T. Daylan et al., The characterization of the gamma-ray signal from the central Milky Way: a case for annihilating dark matter, Phys. Dark Univ. 12 (2016) 1 [arXiv:1402.6703] [INSPIRE].

[11] B. Zhou et al., GeV excess in the Milky Way: the role of diffuse galactic gamma-ray emission templates, Phys. Rev. D 91 (2015) 123010 [arXiv:1406.6948] [INSPIRE].

[12] F. Calore, I. Cholis and C. Weniger, Background model systematics for the Fermi GeV excess, JCAP 03 (2015) 038 [arXiv: 1409.0042] [INSPIRE].

[13] P. Agrawal, B. Batell, P.J. Fox and R. Harnik, WIMPs at the galactic center, JCAP 05 (2015) 011 [arXiv:1411.2592] [INSPIRE].

[14] A. Alves, S. Profumo, F.S. Queiroz and W. Shepherd, Effective field theory approach to the Galactic Center gamma-ray excess, Phys. Rev. D 90 (2014) 115003 [arXiv:1403.5027] [INSPIRE].

[15] M. Duerr, P. Fileviez Perez and J. Smirnov, Gamma-ray excess and the minimal dark matter model, arXiv:1510.07562 [INSPIRE].

[16] J.D. Ruiz-Alvarez, C.A. de S. Pires, F.S. Queiroz, D. Restrepo and P.S. Rodrigues da Silva, On the connection of gamma-rays, dark matter and Higgs searches at LHC, Phys. Rev. D 86 (2012) 075011 [arXiv:1206.5779] [INSPIRE].

[17] J. Cao, L. Shang, P. Wu, J.M. Yang and Y. Zhang, Supersymmetry explanation of the Fermi Galactic Center excess and its test at LHC run II, Phys. Rev. D 91 (2015) 055005 [arXiv: 1410.3239] [INSPIRE].

[18] K. Ghorbani, Fermionic dark matter with pseudo-scalar Yukawa interaction, JCAP 01 (2015) 015 [arXiv: 1408.4929] [INSPIRE].

[19] K. Ghorbani and H. Ghorbani, Scalar split WIMPs in the future direct detection experiments, arXiv: 1501.00206 [INSPIRE].

[20] K. Ghorbani and H. Ghorbani, Two-portal dark matter, Phys. Rev. D 91 (2015) 123541 [arXiv: 1504.03610] [INSPIRE].

[21] Fermi-LAT collaboration, M. Ackermann et al., Constraining Dark Matter models from a combined analysis of Milky Way satellites with the Fermi Large Area Telescope, Phys. Rev. Lett. 107 (2011) 241302 [arXiv:1108.3546] [INSPIRE].

[22] A. Geringer-Sameth and S.M. Koushiappas, Exclusion of canonical WIMPs by the joint analysis of Milky Way dwarfs with Fermi, Phys. Rev. Lett. 107 (2011) 241303 [arXiv: 1108.2914] [INSPIRE].

[23] Y.-L.S. Tsai, Q. Yuan and X. Huang, A generic method to constrain the dark matter model parameters from Fermi observations of dwarf spheroids, JCAP 03 (2013) 018 [arXiv:1212.3990] [INSPIRE]. 
[24] M.N. Mazziotta, F. Loparco, F. de Palma and N. Giglietto, A model-independent analysis of the Fermi Large Area Telescope gamma-ray data from the Milky Way dwarf galaxies and halo to constrain dark matter scenarios, Astropart. Phys. 37 (2012) 26 [arXiv:1203.6731] [INSPIRE].

[25] Fermi-LAT collaboration, M. Ackermann et al., Dark matter constraints from observations of 25 Milky Way satellite galaxies with the Fermi Large Area Telescope, Phys. Rev. D 89 (2014) 042001 [arXiv: 1310.0828] [INSPIRE].

[26] Fermi-LAT collaboration, M. Ackermann et al., Searching for dark matter annihilation from Milky Way dwarf spheroidal galaxies with six years of Fermi Large Area Telescope data, Phys. Rev. Lett. 115 (2015) 231301 [arXiv:1503.02641] [INSPIRE].

[27] A. Geringer-Sameth, S.M. Koushiappas and M.G. Walker, Comprehensive search for dark matter annihilation in dwarf galaxies, Phys. Rev. D 91 (2015) 083535 [arXiv:1410.2242] [INSPIRE].

[28] Fermi-LAT collaboration, M. Wood et al., Dark matter searches with the Fermi-LAT in the direction of dwarf spheroidals, arXiv:1507.03530 [INSPIRE].

[29] A. Geringer-Sameth et al., Indication of gamma-ray emission from the newly discovered dwarf galaxy Reticulum II, Phys. Rev. Lett. 115 (2015) 081101 [arXiv:1503.02320] [INSPIRE].

[30] D. Hooper and T. Linden, On the gamma-ray emission from Reticulum II and other dwarf galaxies, JCAP 09 (2015) 016 [arXiv: 1503.06209] [INSPIRE].

[31] DES, Fermi-LAT collaboration, A. Drlica-Wagner et al., Search for gamma-ray emission from DES dwarf spheroidal galaxy candidates with Fermi-LAT data, Astrophys. J. 809 (2015) L4 [arXiv: 1503.02632] [INSPIRE].

[32] S. Li et al., Search for gamma-ray emission from eight dwarf spheroidal galaxy candidates discovered in year two of dark energy survey with Fermi-LAT data, Phys. Rev. D 93 (2016) 043518 [arXiv: 1511.09252] [INSPIRE].

[33] A. Martin, J. Shelton and J. Unwin, Fitting the Galactic Center gamma-ray excess with cascade annihilations, Phys. Rev. D 90 (2014) 103513 [arXiv:1405.0272] [INSPIRE].

[34] D.G. Cerdeno, M. Peiro and S. Robles, Fits to the Fermi-LAT GeV excess with RH sneutrino dark matter: implications for direct and indirect dark matter searches and the LHC, Phys. Rev. D 91 (2015) 123530 [arXiv:1501.01296] [INSPIRE].

[35] A. Rajaraman, J. Smolinsky and P. Tanedo, On-shell mediators and top-charm dark matter models for the Fermi-LAT galactic center excess, arXiv:1503.05919 [INSPIRE].

[36] M. Abdullah, A. DiFranzo, A. Rajaraman, T.M.P. Tait, P. Tanedo and A.M. Wijangco, Hidden on-shell mediators for the Galactic Center $\gamma$-ray excess, Phys. Rev. D 90 (2014) 035004 [arXiv: 1404.6528] [inSPIRE].

[37] J. Cao, L. Shang, P. Wu, J.M. Yang and Y. Zhang, Interpreting the galactic center gamma-ray excess in the NMSSM, JHEP 10 (2015) 030 [arXiv:1506.06471] [INSPIRE].

[38] A. Berlin, P. Gratia, D. Hooper and S.D. McDermott, Hidden sector dark matter models for the Galactic Center gamma-ray excess, Phys. Rev. D 90 (2014) 015032 [arXiv:1405.5204] [INSPIRE].

[39] T. Gherghetta, B. von Harling, A.D. Medina, M.A. Schmidt and T. Trott, SUSY implications from WIMP annihilation into scalars at the Galactic Center, Phys. Rev. D 91 (2015) 105004 [arXiv: 1502.07173] [INSPIRE]. 
[40] J.M. Cline, G. Dupuis, Z. Liu and W. Xue, Multimediator models for the galactic center gamma ray excess, Phys. Rev. D 91 (2015) 115010 [arXiv:1503.08213] [INSPIRE].

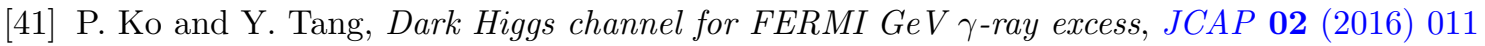
[arXiv: 1504.03908] [INSPIRE].

[42] G. Elor, N.L. Rodd and T.R. Slatyer, Multistep cascade annihilations of dark matter and the Galactic Center excess, Phys. Rev. D 91 (2015) 103531 [arXiv:1503.01773] [InSPIRE].

[43] M. Heikinheimo and C. Spethmann, Galactic Centre GeV photons from dark technicolor, JHEP 12 (2014) 084 [arXiv:1410.4842] [INSPIRE].

[44] C. Boehm, M.J. Dolan and C. McCabe, A weighty interpretation of the Galactic Centre excess, Phys. Rev. D 90 (2014) 023531 [arXiv:1404.4977] [InSPIRE].

[45] D.G. Cerdeno and O. Seto, Right-handed sneutrino dark matter in the NMSSM, JCAP 08 (2009) 032 [arXiv:0903.4677] [INSPIRE].

[46] F. Deppisch and A. Pilaftsis, Thermal right-handed sneutrino dark matter in the $F(D)$-term model of hybrid inflation, JHEP 10 (2008) 080 [arXiv:0808.0490] [INSPIRE].

[47] S.T. Iqbal and Z. Lei, Generally extended MSSM with right-handed neutrinos, J. Phys. G 42 (2015) 095003 [INSPIRE].

[48] W. Wang, J.M. Yang and L.L. You, Higgs boson mass in NMSSM with right-handed neutrino, JHEP 07 (2013) 158 [arXiv:1303.6465] [INSPIRE].

[49] Z. Kang, J. Li, T. Li, T. Liu and J. Yang, Asymmetric sneutrino dark matter in the NMSSM with minimal inverse seesaw, arXiv:1102.5644 [INSPIRE].

[50] D.G. Cerdeno, J.-H. Huh, M. Peiro and O. Seto, Very light right-handed sneutrino dark matter in the NMSSM, JCAP 11 (2011) 027 [arXiv:1108.0978] [INSPIRE].

[51] Y.-L. Tang, One right-handed neutrino to generate complete neutrino mass spectrum in the framework of NMSSM, Nucl. Phys. B 890 (2014) 263 [arXiv:1411.1892] [InSPIRE].

[52] R. Allahverdi, S. Bornhauser, B. Dutta and K. Richardson-McDaniel, Prospects for indirect detection of sneutrino dark matter with IceCube, Phys. Rev. D 80 (2009) 055026 [arXiv: 0907.1486] [INSPIRE].

[53] R. Allahverdi, S. Campbell and B. Dutta, Extragalactic and galactic gamma-rays and neutrinos from annihilating dark matter, Phys. Rev. D 85 (2012) 035004 [arXiv:1110.6660] [INSPIRE].

[54] R. Allahverdi, B. Dutta, K. Richardson-McDaniel and Y. Santoso, Sneutrino dark matter and the observed anomalies in cosmic rays, Phys. Lett. B 677 (2009) 172 [arXiv:0902.3463] [INSPIRE].

[55] R. Allahverdi, S.S. Campbell, B. Dutta and Y. Gao, Dark matter indirect detection signals and the nature of neutrinos in the supersymmetric $U(1)_{B-L}$ extension of the standard model, Phys. Rev. D 90 (2014) 073002 [arXiv: 1405.6253] [INSPIRE].

[56] P. Minkowski, $\mu \rightarrow e \gamma$ at a rate of one out of $10^{9}$ muon decays?, Phys. Lett. B 67 (1977) 421 [INSPIRE].

[57] T. Yanagida, Horizontal symmetry and masses of neutrinos, in the proceedings of the Workshop on unified theory and baryon number in the universe, O. Sawada and A. Sugamoto eds., KEK, Tsukuba, Japan (1979). 
[58] M. Gell-Mann, P. Ramond, R. Slansky, Complex spinors and unified theories, in Supergravity, D.Z. Freedman and P.van Nieuwenhuizen eds., North Holland, Amsterdam, The Netherlands (1979).

[59] S.L. Glashow, The future of elementary particle physics, in Quarks and leptons, Cargèse lectures, M. Lévy et al. eds., Plenum Press, New York, U.S.A. (1980).

[60] R.N. Mohapatra and G. Senjanović, Neutrino mass and spontaneous parity violation, Phys. Rev. Lett. 44 (1980) 912 [INSPIRE].

[61] G. Bélanger, F. Boudjema, A. Pukhov and A. Semenov, MicrOMEGAs4.1: two dark matter candidates, Comput. Phys. Commun. 192 (2015) 322 [arXiv:1407.6129] [INSPIRE].

[62] J. Alwall et al., The automated computation of tree-level and next-to-leading order differential cross sections and their matching to parton shower simulations, JHEP 07 (2014) 079 [arXiv: 1405.0301] [INSPIRE].

[63] T. Sjöstrand et al., An introduction to PYTHIA 8.2, Comput. Phys. Commun. 191 (2015) 159 [arXiv: 1410.3012] [INSPIRE].

[64] A. Alloul, N.D. Christensen, C. Degrande, C. Duhr and B. Fuks, FeynRules 2.0 - A complete toolbox for tree-level phenomenology, Comput. Phys. Commun. 185 (2014) 2250 [arXiv: 1310.1921] [INSPIRE].

[65] Particle Data Group collaboration, K.A. Olive et al., Review of particle physics, Chin. Phys. C 38 (2014) 090001 [INSPIRE].

[66] C.O. Dib and C.S. Kim, Discovering sterile neutrinos ligther than $M_{W}$ at the LHC, Phys. Rev. D 92 (2015) 093009 [arXiv: 1509.05981] [INSPIRE].

[67] ATLAS collaboration, Search for heavy Majorana neutrinos with the ATLAS detector in pp collisions at $\sqrt{s}=8 \mathrm{TeV}$, JHEP 07 (2015) 162 [arXiv:1506.06020] [INSPIRE].

[68] CMS collaboration, Search for heavy Majorana neutrinos in $\mu^{ \pm} \mu^{ \pm}+$jets events in proton-proton collisions at $\sqrt{s}=8 \mathrm{TeV}$, Phys. Lett. B 748 (2015) 144 [arXiv:1501.05566] [INSPIRE].

[69] S. Antusch and O. Fischer, Testing sterile neutrino extensions of the standard model at future lepton colliders, JHEP 05 (2015) 053 [arXiv: 1502.05915] [INSPIRE].

[70] F.F. Deppisch, P.S. Bhupal Dev and A. Pilaftsis, Neutrinos and collider physics, New J. Phys. 17 (2015) 075019 [arXiv: 1502.06541] [INSPIRE].

[71] A. Das, P.S. Bhupal Dev and N. Okada, Direct bounds on electroweak scale pseudo-Dirac neutrinos from $\sqrt{s}=8 \mathrm{TeV}$ LHC data, Phys. Lett. B 735 (2014) 364 [arXiv:1405.0177] [INSPIRE].

[72] S. Antusch and O. Fischer, Non-unitarity of the leptonic mixing matrix: present bounds and future sensitivities, JHEP 10 (2014) 094 [arXiv:1407.6607] [INSPIRE].

[73] A. Das and N. Okada, Improved bounds on the heavy neutrino productions at the LHC, Phys. Rev. D 93 (2016) 033003 [arXiv: 1510.04790] [INSPIRE].

[74] A. Das and N. Okada, Inverse seesaw neutrino signatures at the LHC and ILC, Phys. Rev. D 88 (2013) 113001 [arXiv:1207.3734] [INSPIRE].

[75] D. Wyler and L. Wolfenstein, Massless neutrinos in left-right symmetric models, Nucl. Phys. B 218 (1983) 205 [INSPIRE]. 
[76] R.N. Mohapatra and J.W.F. Valle, Neutrino mass and baryon number nonconservation in superstring models, Phys. Rev. D 34 (1986) 1642 [INSPIRE].

[77] E. Ma, Lepton number nonconservation in $E_{6}$ superstring models, Phys. Lett. B 191 (1987) 287 [INSPIRE].

[78] R.N. Mohapatra, Mechanism for understanding small neutrino mass in superstring theories, Phys. Rev. Lett. 56 (1986) 561 [INSPIRE].

[79] Y.-L. Zhou, Neutrino masses and flavor mixing in a generalized inverse seesaw model with a universal two-zero texture, Phys. Rev. D 86 (2012) 093011 [arXiv: 1205.2303] [INSPIRE].

[80] J.F. Navarro, C.S. Frenk and S.D.M. White, The structure of cold dark matter halos, Astrophys. J. 462 (1996) 563 [astro-ph/9508025] [INSPIRE].

[81] Y.-L.S. Tsai, Q. Yuan and X. Huang, A generic method to constrain the dark matter model parameters from Fermi observations of dwarf spheroids, JCAP 03 (2013) 018 [arXiv: 1212.3990] [INSPIRE].

[82] F. Iocco, M. Pato, G. Bertone and P. Jetzer, Dark matter distribution in the Milky Way: microlensing and dynamical constraints, JCAP 11 (2011) 029 [arXiv:1107.5810] [INSPIRE]. 